\title{
The Flexible Adaptive Optics Concept
}

\author{
Laurent Jolissaint $^{\mathrm{a}}$, Stephen S. Eikenberry ${ }^{\mathrm{b}}$, Audrey Bouxin ${ }^{\mathrm{a}}$, Onur Keskin ${ }^{\mathrm{c}}$, and Cahit \\ Yesilyaprak $^{\mathrm{d}}$ \\ ${ }^{a}$ University of Applied Sciences Western Switzerland, Yverdon-les-Bains, Switzerland \\ ${ }^{\mathrm{b}}$ Department of Astronomy, University of Florida, Gainesville, Fl, USA \\ ${ }^{\mathrm{c}}$ Centre for Optomechatronic Research \& Application (OPAM), Işik University, Istanbul, \\ Turkey \\ ${ }^{\mathrm{d}}$ Ataturk University Astrophysics Research \& Application Center (ATASAM), Erzurum, \\ Turkey
}

\begin{abstract}
The availability of small deformable mirrors with large number of actuators and stroke, on one hand, and versatile wavefront sensors (pyramid WFS), on the other, allows the development of AO systems whose equivalent pitch can be adapted freely to the guide stars and seeing conditions. On moderately large telescopes (4 m class) this flexibility allows a performance (Strehl and limiting magnitude) always better than what a classical, frozen Shack-Hartman design would allow. Moreover, adding several natural guide star wavefront sensors, we believe a single system could do anything from extreme AO to GLAO, i.e using the same hardware, and adapting the control software parameters to the observation mode.

Taking advantage of such a system requires the use of a zoom optics in the imager in order to optimally match the plate scale with the PSF. We demonstrate that sub-optimal pixel size would result in a significant loss in term of science data reduction, in particular object detectability.

The raison d'être of such a versatile system is to popularize AO in community of astronomers not familiar with it, by allowing, on the same telescope, high resolution as well as seeing improved observations. This is particularly important for countries where very few large telescopes are available. On the long term, we think that most moderate size telescopes will have this sort of flexible multi-purpose AO systems as a default. The flexible AO concept will be implemented on the new $4 \mathrm{~m}$ Turkish telescope, DAG.
\end{abstract}

Keywords: DAG observatory; $4 \mathrm{~m}$ class telescopes; adaptive optics; multi-purpose
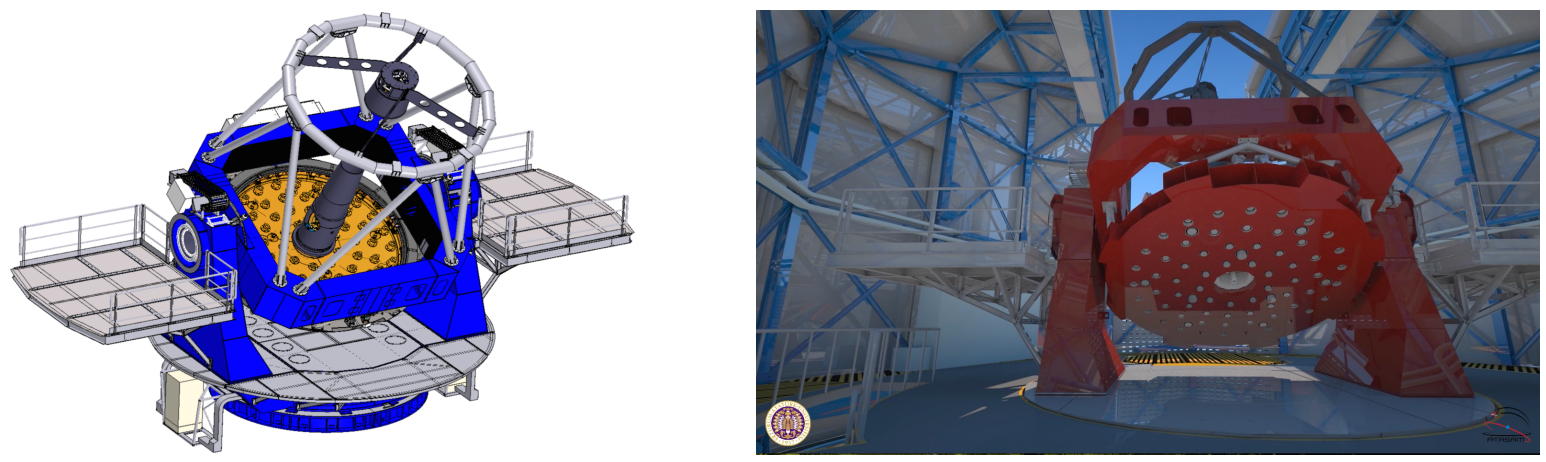

Figure 1. DAG, the new $4 \mathrm{~m}$ Turkish telescope (synthetic images - first light for 2020).

Further author information: e-mail: laurent.jolissaint@heig-vd.ch 


\section{INTRODUCTION}

Atatürk University (Erzurum, Turkey) is developing a new $4 \mathrm{~m}$ astronomical telescope 1 that will be installed on a 3170 m summit near the city of Erzurum, Turkey, in August 2020. DAG (for Dogu Anadolu Gozlemevi, i.e Eastern Anatolia Observatory) will be a multi-purpose telescope, allowing imaging and spectroscopic observations in the visible and infrared domain. One of the Nasmyth platform will be dedicated to seeing limited (SL) observations, the other to $\mathrm{AO}$ aided and SL observations. ${ }^{1}$

Because AO science is new in Turkey, local astronomers do not have much experience in using this technology. Therefore, it was left to the AO team to propose a solution that would be the most versatile as possible, to allow several type of science observation, and initiate the development of AO in Turkey. In short, the AO team had carte blanche.

This opportunity is arriving at an ideal time in the history of AO development : first, many AO concepts have been tested successfully - from extreme to ground layer AO - and second, highly efficient technologies are now available, from miniature high order deformable mirrors to fully adjustable wavefront sensors, and very smart wavefront control schemes. These technological developments were required to make possible AO correction on the future extremely large telescopes. But we do not need to have a $40 \mathrm{~m}$ telescope to make use of them.

Our proposition for DAG AO is to make use of the best technologies currently available and built a multimode AO system, using a single set of hardware components (DM and WFS). Our intention is to built a system that can not only adjust its degree of correction to the seeing and guide star conditions, but also adapt the size of the corrected field-of-view to the available NGS in the observation field.

This versatile AO idea is based on three facts :

1. relatively small deformable mirrors (DM) with large stroke and a large number of actuators are now available in the optics industry;

2. pyramid wavefront sensors (PWFS) efficiency has been clearly demonstrated on several systems $\left(\mathrm{LBT},{ }^{23}\right)$;

3. wavefront reconstruction scheme allows a diversity of choice in the application of the correction, from large field (ground layer) to narrow field (tomography).

Considering these facts, we think the technology is now ready to allow, on moderate size telescopes, multimodes AO systems, from ExAO to GLAO, using the same hardware, in different configurations, similar to what has been tested on the CANARY system. ${ }^{4}$

\section{THE FLEXIBLE AO CONCEPT}

\subsection{Adapting the system's order of correction to the guide star brightness}

Let us consider the case of DAG, a $4 \mathrm{~m}$ telescope. For a moderate 1" seeing, about 80 actuators are required to get a decent correction in the NIR (Strehl about $70 \%$ with a bright NGS, basically one actuator per $r_{0}$ length at the wavelength of observation). Now, let us assume we have a DM with many more actuators, for instance 4 times more, at 320 . We shall build a modal basis for this mirror, adapted to the turbulent spectrum (for instance using Karhunen.-Loeve modes, or the disc harmonics, or some mixture of Zernike modes). The number of modes that we should actually correct (and set the others coefficients to 0) depends on our capability to measure the modes amplitude with some accuracy. Classically, modal control (Gendron et al.) shall be implemented (the lower a mode's SNR, the lower its gain).

If we have a bright NGS, or low seeing or low wind, then we can imagine to exploit more actuators than the nominal SCAO case. If the conditions are less favorable, then on the contrary we would want to lower the number of corrected modes. Modal control can help indeed, but if we use a Shack-Hartmann system, there is no way we could adapt the WFS measurement range to the NGS/seeing conditions.

At this point, it is obvious for anyone that the PWFS can fully lift this limitation. The spatial sampling and sensitivity can be fully adapted to the NGS/seeing conditions to get the best signal/noise ratio for a given observation. At any time, one can select the optimal numbers of modes to compensate, from an equivalent ExAO mode to a GLAO mode. 

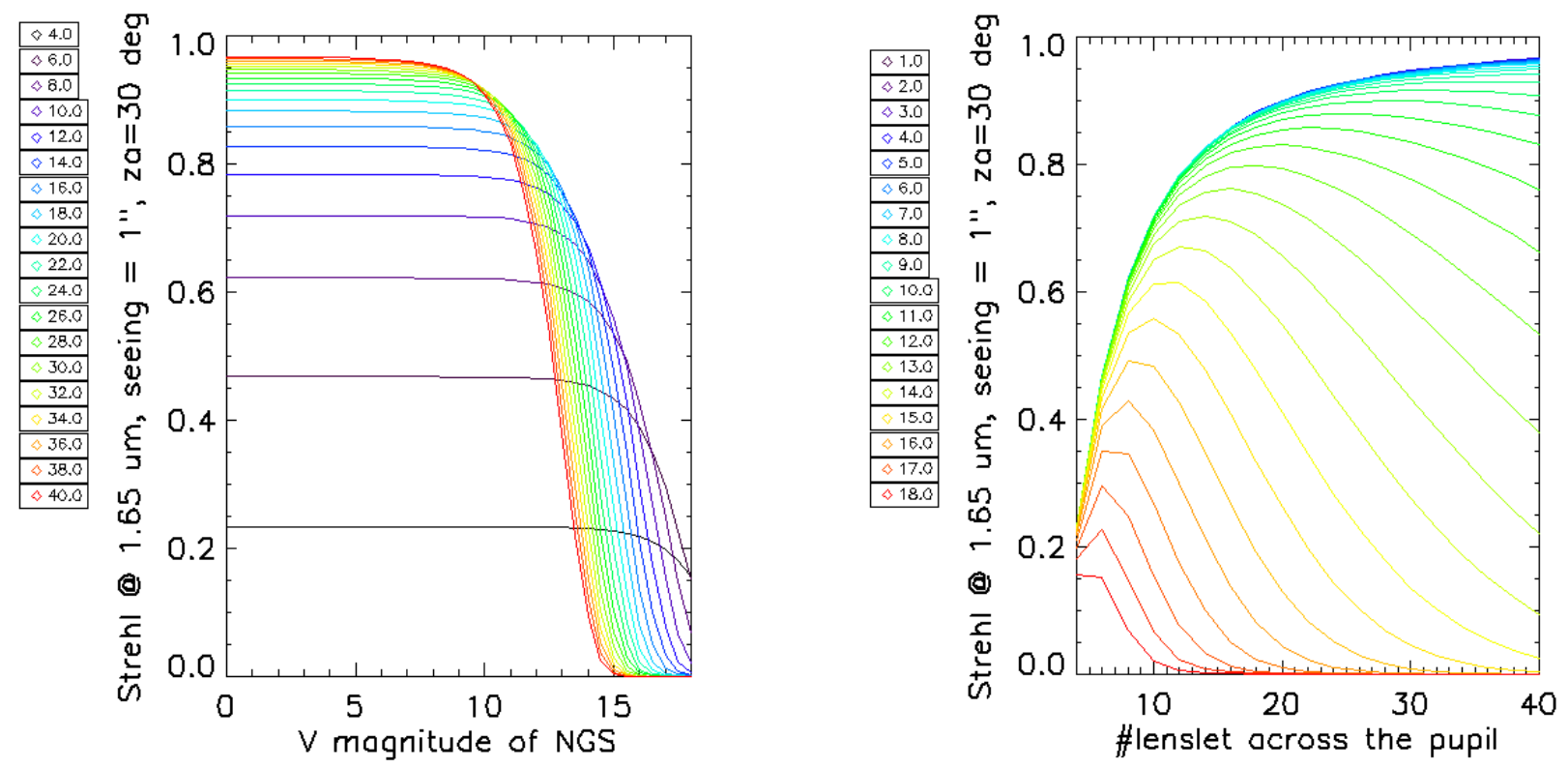

FiguRE 2. Left : Strehl versus NGS magnitude for an increased DM pitch (as indicated at left). Right : Strehl versus number of lenslets across the pupil, for increased NGS magnitudes (as indicated at left).

This is shown in Fig. 2. Finely adjusting the system's order of correction to the NGS magnitude allows us to reach, all the time, the best possible correction. In other words, we can follow the envelope of the Strehl versus magnitude shown in Fig. 2, or, as illustrated in Fig. 2, right, chose the pitch (or equivalently the number of modes) that maximize the Strehl for a given NGS magnitude.

In other words, we believe that by coupling high order DM with PWFS, we can build AO systems able to get the maximum out of the observing conditions.

- if the NGS is bright, the seeing and the wind moderate, we can use all the modes and run in an EXAO mode (300 modes for a $4 \mathrm{~m}$ telescope is indeed EXAO);

- if the NGS gets dimmer, the order of the system is adapted to maximize the Strehl.

In fact, there is no need to separate the AO jargon in ExAO SCAO etc. Rather, we now have a system that is fully versatile, and always adapt itself to the conditions, getting the best possible Strehl, by carefully selecting the number of modes to compensate (basically a gain from 0 to 1 to each mode is applied within a modal control scheme).

According to the figure, a gain of a few magnitudes (up to 2, maybe more) can be expected with respect to the non-optimized case. We are now running detailed Monte-Carlo simulations to explore in details the feasibility of the concept.

\subsection{Adapting the number of NGS and DM command to the science observation type}

Now we introduce the second level of flexibility : the number of NGS we want to use, and how we use the optical turbulence information we get from these stars.

Let us assume we have 4 PWFS, but only one high-order DM. Consider a given science object, around which there is naturally a certain number of stars. Which stars are used and how the DM command is built should depend on the type of science observation and object properties. Many cases are possible :

- the science object is point-like \& bright, it becomes the NGS and we go for an EXAO like type of observation;

- the science object is small but faint, we shall use the nearest NGS, and how many NGS depends on the balance between the angular anisoplanatism error and the noise error; of course each PWFS shall be adjusted to its NGS magnitude; if possible, a tomographic reconstruction can be done and the DM 
command computed in the direction of the science object, as it is done in the Laser Tomography scheme, but here using NGS ;

- the science object is somewhat extended (a cluster of faint structures), then again we might want to use either a tomographic reconstruction and a correction oriented to the center of the object, or a classical $\mathrm{GLAO}^{5}$ scheme, if the object is significantly extended.

Needless to say, for each case, the number of modes to correct (or the modal gains) shall be adapted using the scheme presented in the previous section. It is also important to note that the concept is useful only if we have access to a good estimate of the $C_{N}^{2}$ profile at the time of the observation. An optical turbulence profiler is therefore required at the site. Or the profile is estimated from the WFS data themselves.

Such an optimization can have a significant positive impact on the quality of the correction, or on the sky coverage using NGS. Basically, the idea is to use optimally the useful information available in the field. In turns, it requires an excellent knowledge of the seeing/stars data : but many sensors or algorithms have been developed today by many authors allowing to retrieve these data from the raw observations themselves, allowing a real time optimization of the system.

To illustrate the concept, we show in Fig. 3 the typical AO performance we would get with a 4 NGS GLAO configuration, as a function of the guide stars constellation radius (average distance of the 4 NGS to the science object). This is compared with the performance of a single NGS system for which the NGS off-axis distance to the object is the same as the constellation radius.

We generated 100 random constellations of bright 4 stars, and the science object was always located at the center of the constellation. The constellation radius was increased from 0 to 1'. The seeing was 1". A median Paranal observatory $C_{N}^{2}$ profile was used. We made use of the Fourier method to compute directly the long exposure PSF. ${ }^{6}$ This case is indicative of the obvious interest of using optimally, within a given FoV, all the NGS that are at similar distances to the science object.
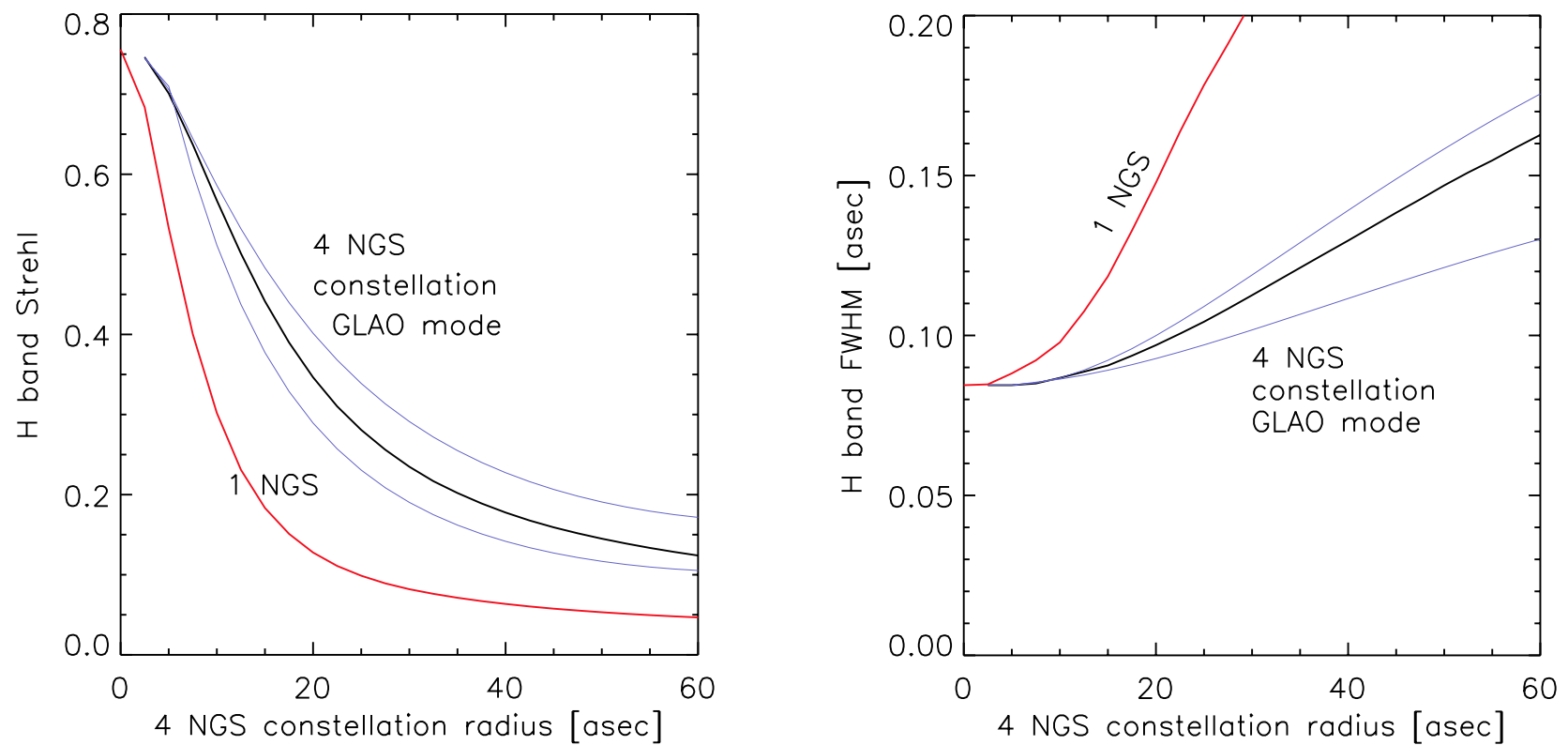

Figure 3. Typical Strehl ratio and PSF FWHM we would get with a random constellation of bright NGS as a function of the average radius of the constellation around the science object.

To conclude, adding a few PWFS to the guide star sensing system, and using smart DM commands computation schemes, we can have an AO that is optimized not only for the quality of the correction, but also for the level of correction required in a given field-of-view. This would increase the diversity of the science program that could benefit from AO correction, using the same system. It is our goal to implement the flexible AO scheme on DAG. 


\section{A ZOOM OPTICS CAMERA TO TAKE ADVANTAGE OF THE FLEXIBLE AO}

Clearly, all PSF sort of sizes have to be expected in the flexible AO concept, from nice circular diffraction limited PSF to potato-shape seeing improved PSF. For DAG, the range of FWHM PSF should be 0.06" to 0.6" (telescope diameter $4 \mathrm{~m}, \mathrm{~J}-\mathrm{H}-\mathrm{K}$ bands). The optimal pixel size for imaging is equal to half of the PSF FWHM (Nyquist sampling). In many systems, the science camera is equipped with a set of 2 or 3 relay optics that can switched and inserted mechanically in the beam to adapt the camera plate scale to the PSF size. But with the large range of FWHM we should expect, this is quite hard to achieve. Beside, a sub-optimal pixel size can have a strong effect on the science data reduction, particularly in the case of crowded star fields, as demonstrated below. Pixel binning is a poor-man's option, but this is not enough to solve the problem.

The solution is to develop a zoom optics. In this case the plate scale can always be adapted to the PSF, taking advantage of the flexible control. In other words, the gain brought by the flexible concept would be wasted if the plate scale could not be optimized.

\subsection{Illustrating the scientific advantage of a zoom optics for AO data reduction}

The key driver for GLAO science instruments is pixel scale. This defines the field of view (FoV) for a given detector size, and also the limiting resolution (which is the key functional parameter of the GLAO system itself). We can define two separate merit functions for the GLAO science camera, based on two different scientific use cases. For the first case, the science driver is basic detection and resolution of point-like sources over the maximum field of view of the camera (the isokinetic patch in the GLAO case). In that case, the "scientific grasp" of the camera (i.e. the number of point sources it can properly study at a given time) can be defined as

$$
\text { Scientific Grasp }=\frac{\text { FoV }^{2}}{\sigma^{2}}
$$

where the FoV is equal to the pixel scale times the number of detector pixels on a side, and $\sigma$ is the Gaussian width for a given GLAO-correction performance $(\mathrm{FWHM} \approx 2.35 \sigma)$, with the requirement for Nyquist-sampling of the PSF (i.e. pixel size is $\leq \sigma$ ). In Fig. 3.1, we show the figure of merit versus GLAO-corrected FWHM for several different cases of fixed pixel scales, and for a zoom camera.

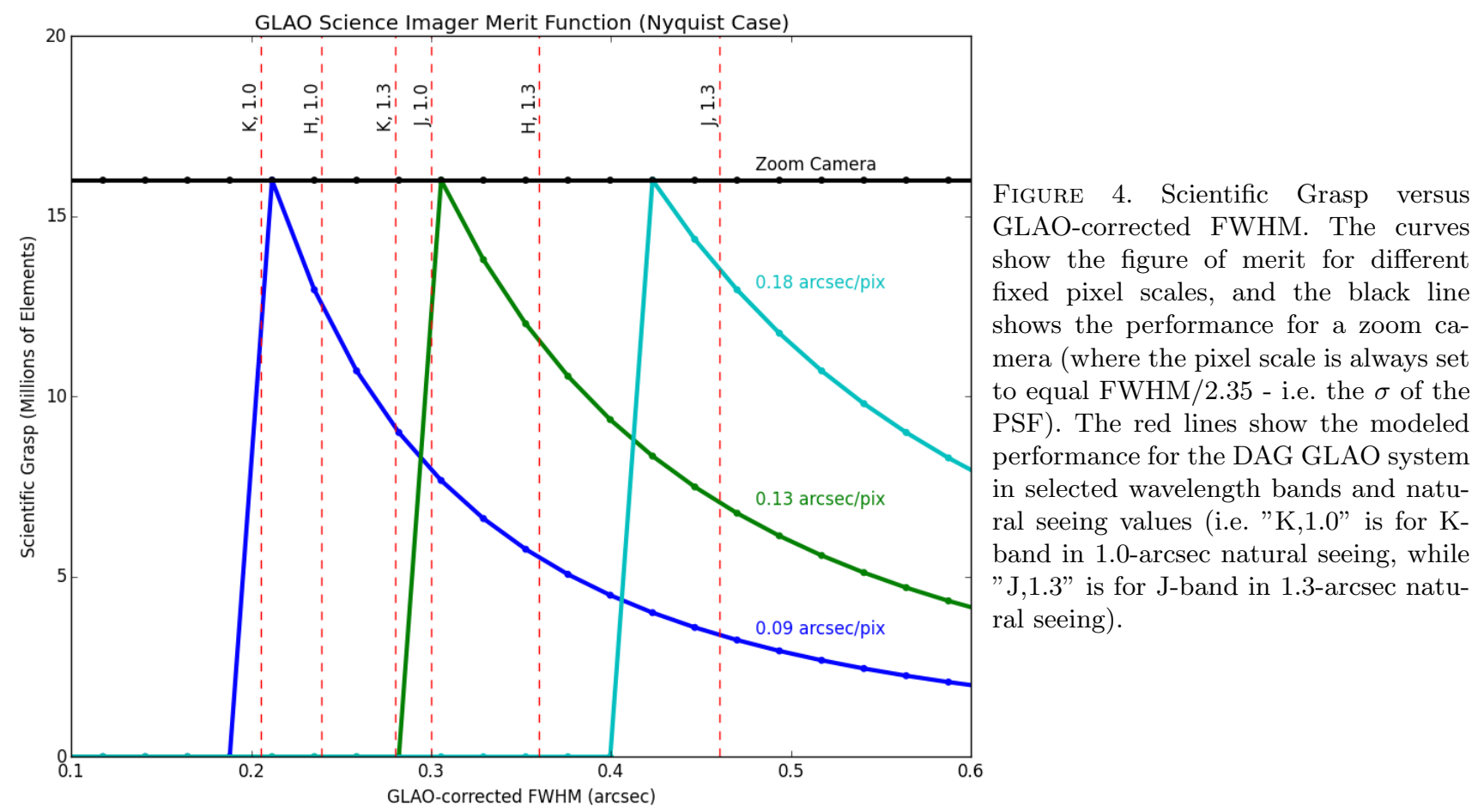


Note that the fixed pixel scales do not provide any functionality for PS $>\sigma$, as they do not Nyquist-sample the PSF. Furthermore, their grasp reaches a maximum value when PS $=\sigma$, but then drops off as $1 / \mathrm{FWHM}^{2}$ as FWHM increases. As a result, a camera with a single pixel scale optimized for the "best" conditions (K-band in 1.0-arcsec seeing) would have optimal performance there, but would drop by more than 5 times in grasp for J-band operation in 1.3-arcsec seeing. Even with the added complexity and expense of 2 or 3 pixel scales, the performance would often drop by factors of more than 2. The zoom camera, on the other hand, is always operating at the maximal efficiency, for any set of observing conditions.

Another common use case is the "super-sampling" mode where the astronomer desires $\mathrm{PS}<\sigma$, in order to provide better PSF reconstruction. This can be driven by a desire for better angular reconstruction of the sources, and/or improved photometry. In that case, we modify the merit function above by multiplying by a function that equals 1 at $\sigma=2 \mathrm{PS}$ (approximately 4.7 pixels per FWHM), and falls off as a sharp Gaussian on the short side (and equals 0 for $\sigma<\mathrm{PS}$ ) and more slowly on the high- $\sigma$ side (representing the loss of $\mathrm{S} / \mathrm{N}$ for spreading out the signal.) We present the results in Fig. 3.1.

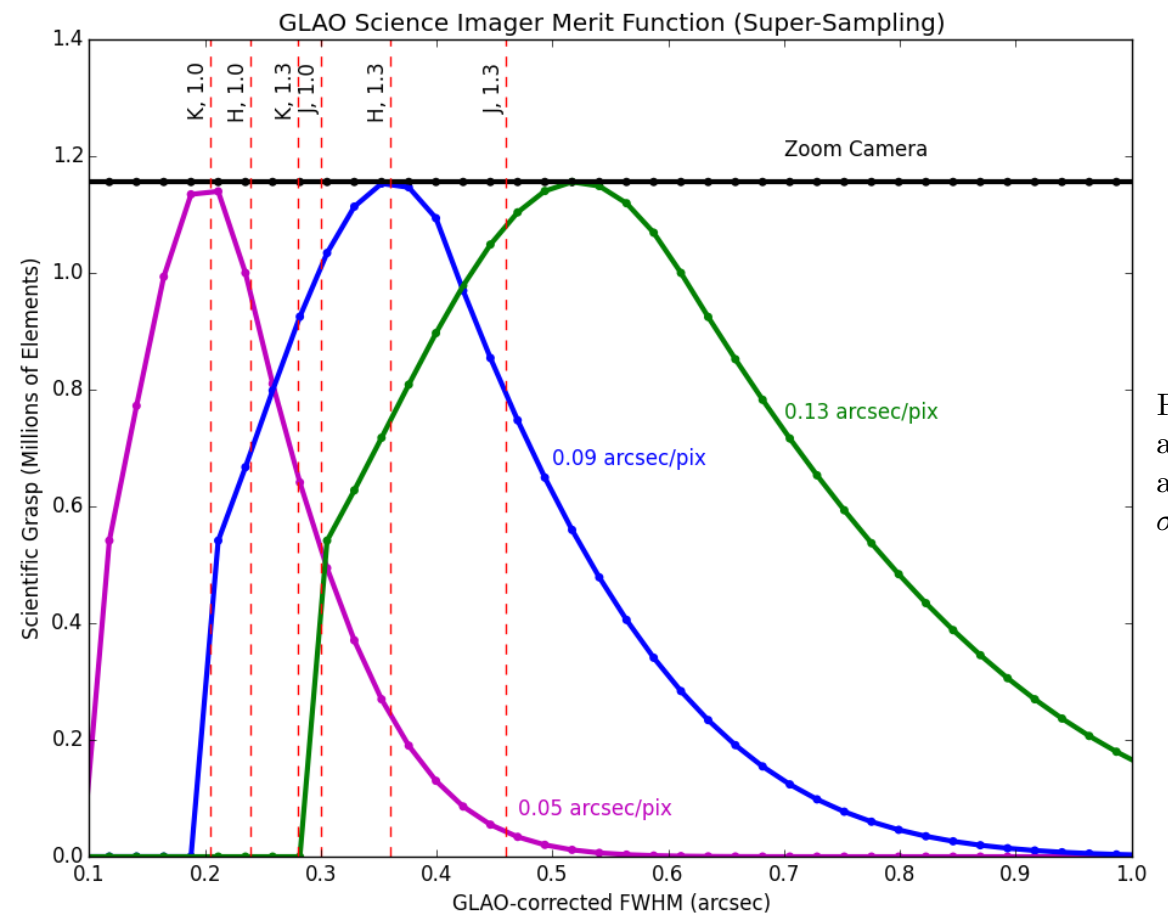

FigURE 5. Super-sampling mode. Same as before except that the pixel scale is always set to FWHM/4.7 - i.e. $1 / 2$ the $\sigma$ of the PSF.

Note that the two finest fixed pixel scales provide limited functionality for much of the GLAO performance space. To achieve better than $50 \%$ of the optimal performance for this sampling case under the best conditions would require yet another fixed pixel scale - and, as before, the performance would often drop by factors of more than 2. The zoom camera, on the other hand, is always operating at the maximal efficiency, for any set of observing conditions.

\section{CONCLUSIONS}

We introduce in this paper the flexible AO concept, for moderate size telescopes. This is not a new mode of operation. Instead, this is a concept where all modes that use a single deformable mirror and 1 or several wavefront sensors are made to work on the same hardware. This new paradigm is made possible thanks to the recent development of high order deformable mirrors and efficient and flexible pyramid wavefront sensors. The objective is to offer a system that has an interest for the largest possible community of astronomers, in particular users of a single telescope. We think such system will become common in the near future on all new moderate size and general purpose telescopes. We demonstrate that to take full advantage of the AO system flexibility zoom optics in the imager are required in order to precisely match the plate scale to the AO PSF. 


\section{ACKNOWLEDGMENTS}

The authors would like to thank Ataturk University, Erzurum/Turkey, Astrophysics Research and Application Center (ATASAM), Erzurum/Turkey ; Republic of Turkey, Ministry of Development ; FMV Isik University, Center of Optomechatronics Application and Research (OPAM), Istanbul/Turkey; and the Haute Ecole d'Ingénierie et de Gestion du Canton de Vaud (HEIG-VD), Yverdon-les-Bains/Switzerland, for their support throughout this research.

\section{REFERENCES}

[1] Jolissaint, L., Keskin, O., Zago, L., Kaan Yerli, S., Yesilyaprak, C., Mudry, E., and Lousberg, G., "The design of an adaptive optics telescope : the case of DAG," in [Ground-based and Airborne Telescopes VI], SPIE 9906, 99063J (July 2016).

[2] Esposito, S., Riccardi, A., Pinna, E., Puglisi, A. T., Quirós-Pacheco, F., Arcidiacono, C., Xompero, M., Briguglio, R., Busoni, L., Fini, L., Argomedo, J., Gherardi, A., Agapito, G., Brusa, G., Miller, D. L., Guerra Ramon, J. C., Boutsia, K., and Stefanini, P., "Natural guide star adaptive optics systems at LBT : FLAO commissioning and science operations status," in [Adaptive Optics Systems III], SPIE 8447, 84470U (July 2012).

[3] Pinna, E., Esposito, S., Hinz, P., Agapito, G., Bonaglia, M., Puglisi, A., Xompero, M., Riccardi, A., Briguglio, R., Arcidiacono, C., Carbonaro, L., Fini, L., Montoya, M., and Durney, O., "SOUL : the Single conjugated adaptive Optics Upgrade for LBT," in [Adaptive Optics Systems V], SPIE 9909, 99093V (July 2016).

[4] Gendron, E., Morris, T., Basden, A., Vidal, F., Atkinson, D., Bitenc, U., Buey, T., Chemla, F., Cohen, M., Dickson, C., Dipper, N., Feautrier, P., Gach, J.-L., Gratadour, D., Henry, D., Huet, J.-M., Morel, C., Morris, S., Myers, R., Osborn, J., Perret, D., Reeves, A., Rousset, G., Sevin, A., Stadler, E., Talbot, G., Todd, S., and Younger, E., "Final two-stage MOAO on-sky demonstration with CANARY," in [Adaptive Optics Systems V], SPIE 9909, 99090C (July 2016).

[5] Rigaut, F., "Ground-Conjugate Wide Field Adaptive Optics for the ELTs," in [Beyond Conventional Adaptive Optics], Vernet, E., Ragazzoni, R., Esposito, S., and Hubin, N., eds., ESO Conference $\mathcal{G}^{3}$ Workshop Proceedings 58, 11-16 (2001).

[6] Jolissaint, L., "Synthetic Modeling of Astronomical Closed Loop Adaptive Optics," Journal of the European Optical Society - Rapid Publications 5 (November 2010). 